\title{
Multivariate linear parametric models applied to daily rainfall time series
}

\author{
S. Grimaldi ${ }^{1}$, F. Serinaldi ${ }^{2}$, and C. Tallerini ${ }^{2}$ \\ ${ }^{1}$ Institute of Research for Hydrogeological Protection, CNR-IRPI, Perugia Italy \\ ${ }^{2}$ Department of Hydraulics, Transportations and Highways, University of Rome "La Sapienza", Rome, Italy
}

Received: 18 November 2004 - Revised: 15 February 2005 - Accepted: 4 March 2005 - Published: 31 March 2005

\begin{abstract}
The aim of this paper is to test the Multivariate Linear Parametric Models applied to daily rainfall series. These simple models allow to generate synthetic series preserving both the time correlation (autocorrelation) and the space correlation (crosscorrelation). To have synthetic daily series, in such a way realistic and usable, it is necessary the application of a corrective procedure, removing negative values and enforcing the no-rain probability. The following study compares some linear models each other and points out the roles of autoregressive (AR) and moving average (MA) components as well as parameter orders and mixed parameters.
\end{abstract}

\section{Introduction}

Daily synthetic series are used in several hydrological applications. In many cases the univariate analysis is not enough, since rainfall series are affected by strong space correlation and a weak time correlation as well. Therefore in a rainfall-scenario simulation the multivariate approach is necessary. In this paper, Multivariate Linear Parametric Models (MLPM) are applied as an extension of the well known Linear Parametric Models (LPM) (Grimaldi, 2004).

Rainfall series are particularly difficult to model with a LPM. Usually they are not perfectly linear, non-Gaussian, and present weak seasonality and a high percentage of zero values (no-rain days). Despite those limits, simulations obtained with LPM preserve the main statistical characteristics of the observed series (Grimaldi et al., 2004). The main problem is the presence of negative values in the synthetic series, an obvious consequence of stochastic nature of these processes that cannot reproduce a sequence of zero-values. In order to overcome this limit we referred to the corrective procedure, already applied in Grimaldi et al. (2004), on 20 daily rainfall series.

Correspondence to: S. Grimaldi

(salvatore.grimaldi@irpi.cnr.it)
Here follows comparisons among MLPMs. The purpose is to point out differences among simple and widely used first-order Vector Autoregressive models, optimal-order Vector Autoregressive models and general Vector Autoregressive Moving Average models described in Sect. 2. The present case study, Sect. 3, also examines the possibility to reduce the number of the parameters in the modelling.

\section{Multivariate linear parametric models}

A multivariate stochastic process can be described by variables characterized by the autocorrelation, in time domain, and the crosscorrelation in the space-time domain. As in the univariate case, these correlations can be expressed by means of parameter linear combinations. The general class of multivariate linear parametric model is called $\operatorname{VARMA}(\mathrm{p}, \mathrm{q})$ (Vector Autoregressive Moving Average, Hall and Nicholls, 1979; Lutkepohl, 1993; Hipel and McLeod, 1994):

$$
\begin{aligned}
& \boldsymbol{y}_{t}=\boldsymbol{v}+\mathbf{A}_{1} \boldsymbol{y}_{t-1}+\mathbf{A}_{2} \boldsymbol{y}_{t-2}+\ldots \ldots \ldots+\mathbf{A}_{p} \boldsymbol{y}_{t-p}+\boldsymbol{u}_{t} \\
& +\mathbf{M}_{1} \boldsymbol{u}_{t-1}+\mathbf{M}_{2} \boldsymbol{u}_{t-2}+\ldots+\mathbf{M}_{q} \boldsymbol{u}_{t-q}
\end{aligned}
$$

where $\boldsymbol{y}_{t}=\left\{y_{1 t}, y_{2 t}, \ldots, y_{k t}\right\}$ is $k$-dimension vector of variables at the time $t, \boldsymbol{v}=\left\{v_{1}, v_{2}, \ldots, v_{k}\right\}$ is a constant vector, $\boldsymbol{u}_{t}=\left\{u_{1 t}, u_{2 t}, \ldots, u_{k t}\right\}$ white-noise vector, and where

$$
\begin{aligned}
& \mathbf{A}_{i}=\left|\begin{array}{l}
a_{i 11} a_{i 12 \ldots \ldots \ldots} a_{i 1 k} \\
a_{i 21} a_{i 22 \ldots \ldots \ldots} a_{i 2 k} \\
\ldots \ldots \ldots \ldots \ldots \ldots . \ldots \\
a_{i k 1} a_{i k 2 \ldots \ldots \ldots} \ldots \ldots
\end{array}\right| i=1,2, \ldots, p,
\end{aligned}
$$

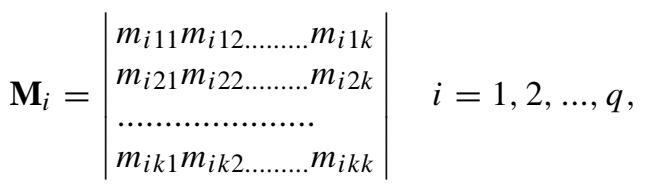

are respectively the Autoregressive and the Moving average coefficient matrices. Since this general expression is usually characterized by a high number of parameters and a complex 

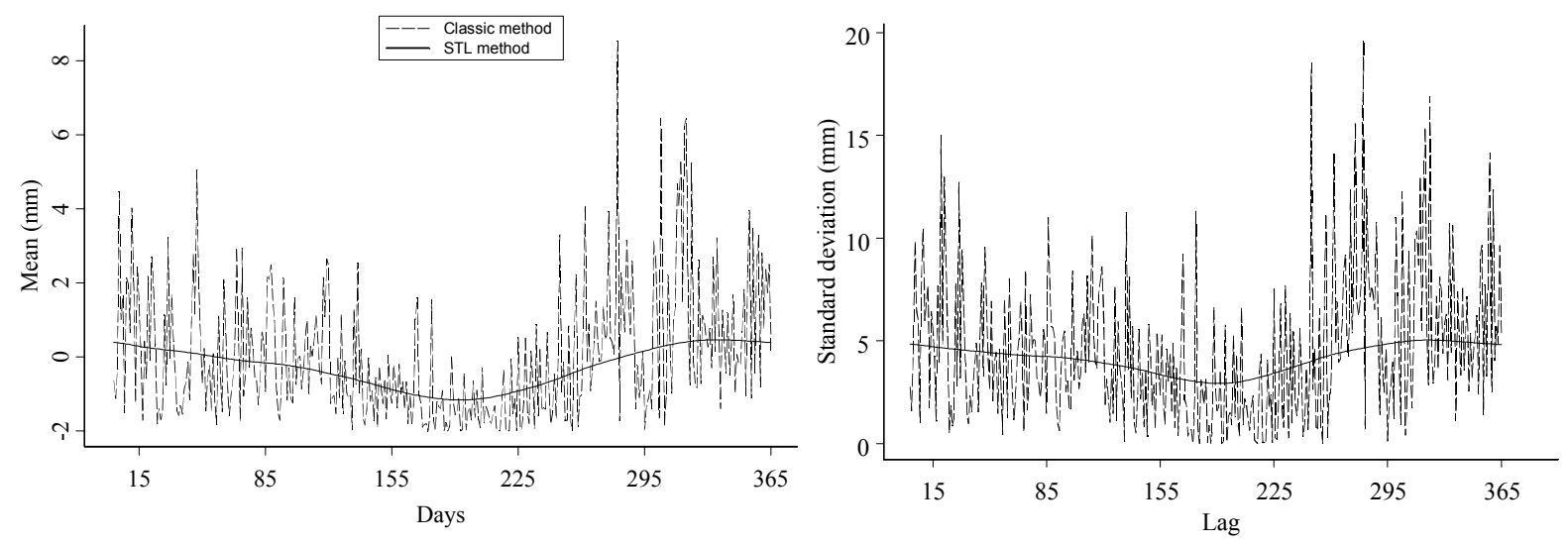

Fig. 1. Seasonal components of series 1 with classic method and STL method applied with smoothing windows of 175 lags.
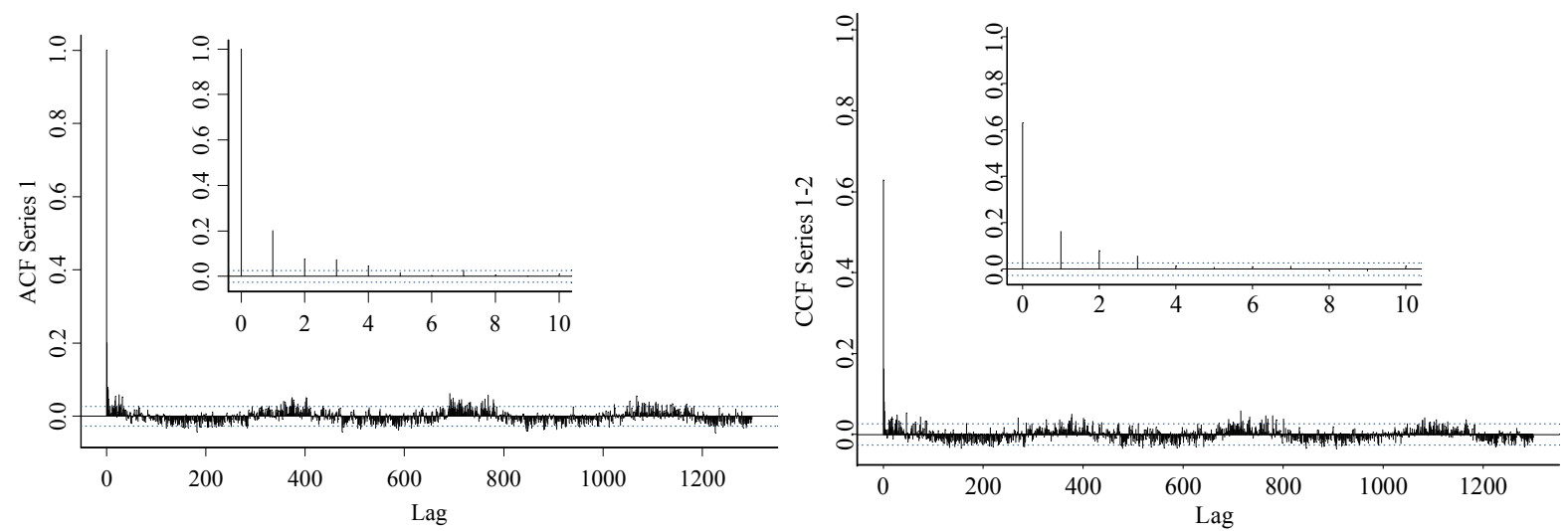

Fig. 2. Autocorrelation Function of series 1 (a) and Cross Correlation Function of series 1-2 (b). The small graph reproduces the above functions until 10 lags.

parameter estimation procedure, in literature it is often simplified. The most used expression is the $\operatorname{VAR}(\mathrm{p})$ easy to be built, which develops only the AR component. Sometimes, even only the first order is considered, VAR(1). Another simplified version is the Contemporaneous $\operatorname{ARMA}(\mathrm{p}, \mathrm{q})$ models (CARMA, Hypel and McLeod, 1994), where all the mixed parameters of both matrices $\mathbf{A}$ and $\mathbf{M}$ are fixed to zero. This means that the model can preserve the full autocorrelation, but the crosscorrelation only at lag 0 ; so rainfall simulation will reproduce only the contemporaneous space correlation.

The procedure to build an MLPM is the same as the univariate case (Grimaldi, 2004). It consists of 4 steps (Preliminary Analysis, Parameter estimation, Checking and Optimal model choice, Simulation) useful to identify the best model analysing the observed data and generate synthetic scenarios. The Preliminary analysis tests the Gaussianity and stationarity on the series. In the first case the Box and Cox transformations are applied, but this approach could prove to be not useful to daily rainfall modelling (Grimaldi, 2004). Concerning the Stationarity, the seasonal characteristics are analysed. The Deseasonalization of single series can be performed fil- tering series $y_{i t}$ with the expression

$z_{i t}^{\tau}=\frac{y_{i t}^{\tau}-\mu_{i \tau}}{s_{i \tau}} \quad i=1,2, \ldots, k$.

The classic mean $\left(\mu_{i \tau}\right)$ and the standard deviation $\left(s_{i \tau}\right)$ periodical components are smoothed using the STL method (Seasonal trend decomposition based on Loess, Grimaldi, 2004). As shown in Fig. 1 this approach is necessary to remove the high noise due to the daily series scale. In daily rainfall series the seasonality could be not significant (Grimaldi et al., 2004, Grimaldi et al., 2004). The Parameter Estimation step uses several methods, such as Yule-Walker (Whittle, 1963), Burg algorithm (Trinidade et al., 2002), OLS (Ordinary Least Square, Lutkepohl, 1993), and MLE (Maximun Likehood Estimation, Shea 1989; Lutkephol, 1993). The following case study uses Lutkephol's (Approximate) MLE method. Together with OLS estimation, there are also methods to reduce the number of the parameters to be estimated, and obtain the so-called Subset VAR models (Lutkephol, 1993). The Checking and Optimal model choice step must check that, for all estimated models, all the residual series are white noises. This is possible through Portmanteau 

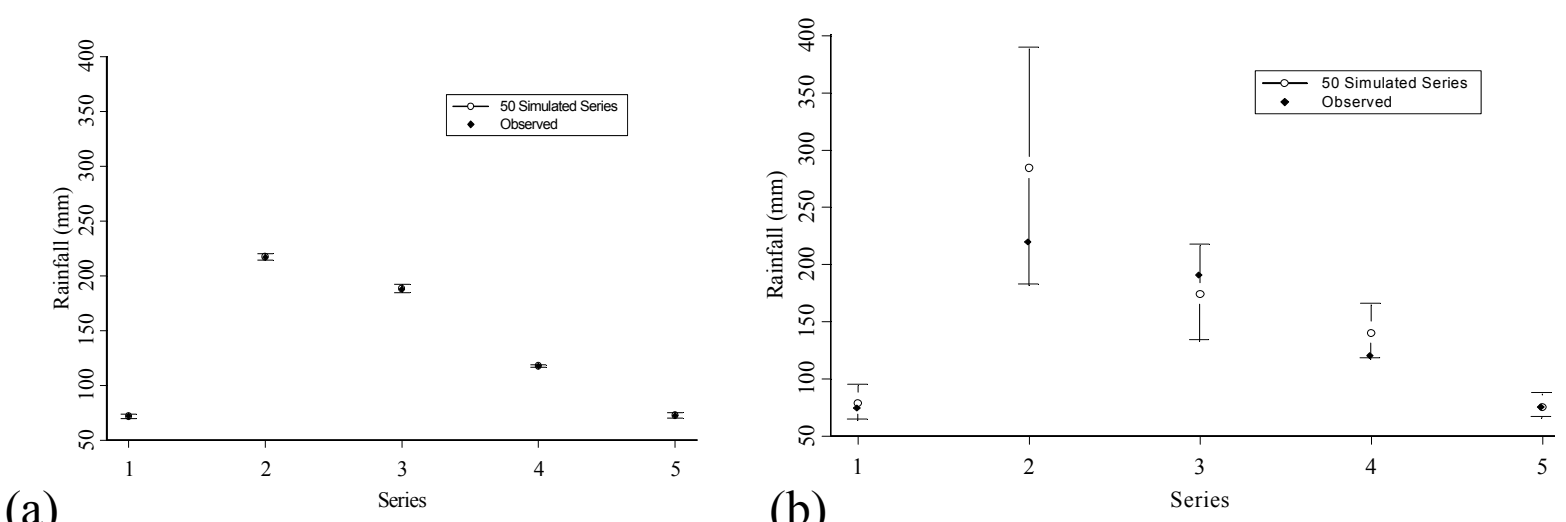

Fig. 3. Maximum values of the observed series and of the 50 simulated series. The box-plot represents the mean of the maximum values and the $5 \%$ and $95 \%$ quantiles estimated on the 50 synthetic values. The results are obtained: (a) without the deseasonalization procedure (b) with the deseasonalization procedure.

Test (Hosking, 1980). Among models positive to this test, the best one is selected by Automatic Selection Index like AIC (Akaike Information Criterion) or SBC (Schwartz Bayesian Criterion). The last step, Simulation, allows to generate synthetic series starting from the selected optimal model. The used simulation algorithm is explained in Lutkepohl (1993) and, as in the univariate case (Grimaldi, 2004), the generation of innovations is carried out by re-sampling the residuals obtained from the observed series. The multivariate case develops a vectorial sampling, so that the contemporaneous correlation is preserved.

As introduced in Sect. 1, these models create negative values in synthetic series, despite their capability to reproduce the main statistics of the observed series. In order to overcome this difficulty, the above-mentioned corrective procedure is necessary. Briefly, this procedure consists in: (i) transferring the abscissa until the negative-value frequency of the simulated series is equal to the no-rain frequency, (ii) changing the negative values in zero-values; (iii) increasing rainy-days values so that enforcing the mean of the observed series. This procedure does not modify the positive tail of the distribution and make the synthetic series realistic and usable.

\section{The case study}

The present case study analyses five daily rainfall series from 1958 to 1979 (Rudari, 2001) observed in some stations of Tuscany, a region of Italy . The examined series originally were lacking in some values and years. The case-study sample is obtained removing the years without values, interpolating missing values and removing 29 February. The final sample consists of a lap of 15 years. Figure 2 shows the autocorrelation (ACF) and a crosscorrelation functions (CCF) of one of the series. As expected there is a weak time correlation and a significant space correlation as well as a very weak seasonal state.
On the 5 described series the following tests are developed:

1. A comparison between the modelling with the deseasonalization procedure (smoothing window $=175$ ) and the modelling without it.

2. A comparison among different models: $\operatorname{Var}(1)$, the simplest and the most used, the optimal $\operatorname{Var}(\mathrm{p})$ and the optimal mixed $\operatorname{Varma}(\mathrm{p}, \mathrm{q})$

3. A comparison between models with different number of parameters: Complete $\operatorname{Var}(\mathrm{p})$ and Subset $\operatorname{Var}(\mathrm{p})$ with the lowest possible number of parameters.

The evaluations were carried out comparing 5 groups of 50 synthetic series, simulated with the different models or procedures, to those observed ones.

Applying on the 5 series the procedure briefly described in Sect. 2, both AIC and SBC index suggest Var(2) as optimal model among Vector AR models, and Varma $(1,1)$ among Vector ARMA models, in particular Portmanteau Test is performed with maximum lag=20 and 5\% significant level.

Firstly the test (a) is approached. The seasonal components of considered series are very weak (see Figs. 1 and 2) and in fact, looking at the ACF (Fig. 2), the sinusoid with period 365 is almost within the $95 \%$ confidence bounds. Figure 3 compares maximum values of the simulated series with and without the application of the deseasonalization procedure and the observed maximum values. It without significant seasonality, the inversion of filter (2) increase the variance of simulated series. 
Table 1a. Statistical parameters estimated on observed and 50 simulated series.

\begin{tabular}{|c|c|c|c|c|c|c|c|c|}
\hline & $\begin{array}{c}\text { mean } \\
\mu\end{array}$ & $\begin{array}{c}\text { mean } \\
\sigma\end{array}$ & $\begin{array}{c}\text { variance } \\
\qquad \mu\end{array}$ & $\begin{array}{c}\text { variance } \\
\sigma\end{array}$ & $\begin{array}{c}\text { skewness } \\
\mu\end{array}$ & $\begin{array}{c}\text { skewness } \\
\sigma\end{array}$ & $\begin{array}{c}\max \text { value } \\
\mu\end{array}$ & $\begin{array}{c}\max \text { value } \\
\sigma\end{array}$ \\
\hline 1 & 2,00 & & 33,51 & & 4,70 & & 72,00 & \\
\hline 2 & 1,86 & & 41,45 & & 10,54 & & 217,00 & \\
\hline 3 & 2,84 & & 68,49 & & 6,27 & & 187,90 & \\
\hline 4 & 2,42 & & 42,05 & & 5,01 & & 117,80 & \\
\hline 5 & 2,38 & & 37,56 & & 4,26 & & 72,60 & \\
\hline \multicolumn{9}{|c|}{5 observed series } \\
\hline 1 & 2,00 & 0,00 & 30,93 & 0,03 & 4,78 & 0,00 & 72,02 & 1,55 \\
\hline 2 & 1,86 & 0,00 & 38,72 & 0,02 & 11,20 & 0,01 & 217,09 & 1,60 \\
\hline 3 & 2,84 & 0,00 & 64,58 & 0,07 & 6,56 & 0,00 & 187,88 & 0,78 \\
\hline 4 & 2,42 & 0,00 & 39,75 & 0,04 & 5,18 & 0,00 & 117,67 & 0,51 \\
\hline 5 & 2,38 & 0,00 & 34,47 & 0,03 & 4,37 & 0,00 & 72,65 & 0,79 \\
\hline \multicolumn{9}{|c|}{5 groups of 50 simulated series with a SVAR(1) } \\
\hline 1 & 2,00 & 0,00 & 30,57 & 0,03 & 4,81 & 0,00 & 72,17 & 1,45 \\
\hline 2 & 1,86 & 0,00 & 38,71 & 0,02 & 11,19 & 0,01 & 216,90 & 1,09 \\
\hline 3 & 2,84 & 0,00 & 64,19 & 0,10 & 6,61 & 0,00 & 187,92 & 2,01 \\
\hline 4 & 2,42 & 0,00 & 39,43 & 0,03 & 5,22 & 0,00 & 117,83 & 0,88 \\
\hline 5 & 2,38 & 0,00 & 34,13 & 0,04 & 4,39 & 0,00 & 72,60 & 0,87 \\
\hline \multicolumn{9}{|c|}{5 groups of 50 simulated series with a SVAR(2) } \\
\hline 1 & 2,00 & 0,00 & 30,07 & 0,09 & 4,85 & 0,00 & 71,76 & 1,43 \\
\hline 2 & 1,86 & 0,00 & 38,46 & 0,06 & 11,29 & 0,01 & 217,25 & 2,67 \\
\hline 3 & 2,84 & 0,00 & 63,82 & 0,07 & 6,65 & 0,00 & 188,19 & 5,34 \\
\hline 4 & 2,42 & 0,00 & 38,90 & 0,04 & 5,26 & 0,00 & 117,71 & 0,61 \\
\hline 5 & 2,38 & 0,00 & 33,61 & 0,07 & 4,42 & 0,00 & 72,72 & 2,27 \\
\hline \multicolumn{9}{|c|}{5 groups of 50 simulated series with a VARMA(1,1) } \\
\hline 1 & 2,00 & 0,00 & 30,42 & 0,03 & 4,82 & 0,00 & 71,82 & 0,64 \\
\hline 2 & 1,86 & 0,00 & 38,51 & 0,04 & 11,23 & 0,00 & 216,78 & 0,65 \\
\hline 3 & 2,84 & 0,00 & 63,90 & 0,07 & 6,62 & 0,00 & 188,02 & 2,44 \\
\hline 4 & 2,42 & 0,00 & 39,12 & 0,04 & 5,23 & 0,00 & 117,74 & 0,77 \\
\hline 5 & 2,38 & 0,00 & 33,91 & 0,03 & 4,40 & 0,00 & 72,85 & 1,81 \\
\hline \multicolumn{9}{|c|}{5 groups of 50 simulated series with a VAR(1) } \\
\hline 1 & 2,00 & 0,00 & 30,19 & 0,04 & 4,84 & 0,00 & 72,28 & 2,23 \\
\hline 2 & 1,86 & 0,00 & 38,45 & 0,04 & 11,25 & 0,01 & 216,89 & 1,34 \\
\hline 3 & 2,84 & 0,00 & 63,74 & 0,10 & 6,64 & 0,00 & 187,69 & 2,03 \\
\hline 4 & 2,42 & 0,00 & 39,05 & 0,04 & 5,25 & 0,00 & 117,98 & 1,69 \\
\hline 5 & 2,38 & 0,00 & 33,79 & 0,05 & 4,44 & 0,00 & 73,48 & 5,13 \\
\hline
\end{tabular}

In order to compare the simulated and the observed series in the tests (a) and (b) the following parameters are mainly investigated: mean, variance, skewness, maximum values, the sum of the first 5 steps of the ACF, rainfall with 50-, 100-, 200-year return time (defined by standard extreme value analysis using Gumbel distribution), wet-dry period transition frequency (frequency of $n$ consecutive rainy days, followed and preceded by dry days), distributions on cumulative rainfall of events of length $n$-days, crosscorrelation function.

Usually the model used in literature is the $\operatorname{Var}(1)$. In this study the optimal model improvements are tested (Var(2) and Varma(1,1)) without deseasonalization procedure. The Table 1 compares the above-described statistical parameters , estimated on the observed series and the 50 simulated ones, Fig. 4 shows an example of comparison between CCF of the simulated series and the observed series functions, that is characteristic for all the simulated series; Fig. 5 shows the
Table 1b. Statistical parameters estimated on observed and 50 simulated series.

\begin{tabular}{|c|c|c|c|c|c|c|c|c|}
\hline & $\begin{array}{c}\text { Sum of first } \\
5 \text { ACF lags } \\
\mu\end{array}$ & $\begin{array}{c}\text { Sum of first } \\
5 \text { ACF lags } \\
\sigma\end{array}$ & $\begin{array}{c}\mathrm{h} \text { with } \\
\mathrm{Tr}=50 \\
\mu\end{array}$ & $\begin{array}{c}\mathrm{h} \text { with } \\
\operatorname{Tr}=50 \\
\sigma\end{array}$ & $\begin{array}{c}\mathrm{h} \text { with } \\
\mathrm{Tr}=100 \\
\mu\end{array}$ & $\begin{array}{c}\mathrm{h} \text { with } \\
\mathrm{Tr}=100 \\
\sigma\end{array}$ & $\begin{array}{c}\mathrm{h} \text { with } \\
\mathrm{Tr}=200 \\
\mu\end{array}$ & $\begin{array}{c}\mathrm{h} \text { with } \\
\mathrm{Tr}=200 \\
\sigma\end{array}$ \\
\hline 1 & 0,05 & & 76,81 & & 82,56 & & 88,30 & \\
\hline 2 & 0,03 & & 181,02 & & 206,00 & & 230,89 & \\
\hline 3 & 0,02 & & 177,99 & & 199,05 & & 220,03 & \\
\hline 4 & 0,03 & & 116,72 & & 129,65 & & 142,54 & \\
\hline 5 & 0,05 & & 80,82 & & 87,63 & & 94,41 & \\
\hline \multicolumn{9}{|c|}{5 observed series } \\
\hline 1 & 0,03 & $3,2 \mathrm{E}-05$ & 77,41 & 3,87 & 83,70 & 5,78 & 89,98 & 8,16 \\
\hline 2 & 0,01 & $7,9 \mathrm{E}-06$ & 179,46 & 2,44 & 204,44 & 3,29 & 229,33 & 4,36 \\
\hline 3 & 0,01 & $6,1 \mathrm{E}-06$ & 174,44 & 9,01 & 195,05 & 11,51 & 215,59 & 14,85 \\
\hline 4 & 0,01 & $3,6 \mathrm{E}-06$ & 112,86 & 3,68 & 124,91 & 5,02 & 136,92 & 6,70 \\
\hline 5 & 0,01 & $1,0 \mathrm{E}-05$ & 81,37 & 5,23 & 88,25 & 7,77 & 95,09 & 10,95 \\
\hline \multicolumn{9}{|c|}{5 groups of 50 simulated series with a SVAR(1) } \\
\hline 1 & 0,03 & $3,3 \mathrm{E}-05$ & 76,99 & 3,48 & 83,15 & 5,73 & 89,29 & 8,64 \\
\hline 2 & 0,01 & $1,1 \mathrm{E}-05$ & 179,92 & 0,89 & 205,01 & 1,48 & 230,01 & 2,36 \\
\hline 3 & 0,01 & $8,0 \mathrm{E}-06$ & 175,00 & 9,18 & 195,78 & 12,70 & 216,49 & 17,13 \\
\hline 4 & 0,02 & $1,7 \mathrm{E}-05$ & 112,28 & 5,45 & 124,14 & 7,86 & 135,97 & 10,84 \\
\hline 5 & 0,02 & $1,7 \mathrm{E}-05$ & 82,03 & 4,82 & 89,05 & 6,96 & 96,05 & 9,64 \\
\hline \multicolumn{9}{|c|}{5 groups of 50 simulated series with a SVAR(2) } \\
\hline 1 & 0,03 & 4,1E-05 & 76,87 & 4,49 & 83,07 & 6,38 & 89,25 & 8,75 \\
\hline 2 & 0,02 & $2,7 \mathrm{E}-05$ & 179,64 & 1,89 & 204,76 & 2,42 & 229,78 & 3,18 \\
\hline 3 & 0,02 & $1,7 \mathrm{E}-05$ & 174,80 & 11,68 & 195,54 & 15,95 & 216,20 & 21,36 \\
\hline 4 & 0,02 & $2,1 \mathrm{E}-05$ & 112,35 & 2,80 & 124,32 & 3,96 & 136,24 & 5,47 \\
\hline 5 & 0,03 & $3,2 \mathrm{E}-05$ & 81,68 & 4,46 & 88,63 & 6,17 & 95,55 & 8,31 \\
\hline \multicolumn{9}{|c|}{5 groups of 50 simulated series with a $\operatorname{VARMA}(1,1)$} \\
\hline 1 & 0,03 & 2,3E-05 & 77,17 & 4,14 & 83,38 & 6,58 & 89,56 & 9,67 \\
\hline 2 & 0,01 & 7,3E-06 & 179,55 & 0,89 & 204,60 & 1,35 & 229,56 & 2,05 \\
\hline 3 & 0,01 & $1,2 \mathrm{E}-05$ & 174,99 & 4,32 & 195,77 & 6,11 & 216,48 & 8,60 \\
\hline 4 & 0,02 & $2,5 \mathrm{E}-05$ & 112,65 & 2,60 & 124,64 & 3,87 & 136,58 & 5,58 \\
\hline 5 & 0,03 & $1,7 \mathrm{E}-05$ & 81,77 & 3,77 & 88,67 & 5,62 & 95,54 & 7,99 \\
\hline \multicolumn{9}{|c|}{5 groups of 50 simulated series with a VAR(1) } \\
\hline 1 & 0,03 & 3,9E-05 & 77,14 & 3,47 & 83,39 & 5,34 & 89,61 & 7,72 \\
\hline 2 & 0,02 & $1,3 \mathrm{E}-05$ & 179,13 & 2,29 & 204,14 & 2,84 & 229,06 & 3,60 \\
\hline 3 & 0,01 & $1,1 \mathrm{E}-05$ & 174,22 & 13,51 & 194,81 & 17,30 & 215,32 & 22,00 \\
\hline 4 & 0,02 & 2,4E-05 & 112,57 & 4,89 & 124,60 & 6,78 & 136,60 & 9,18 \\
\hline 5 & 0,03 & $4,2 \mathrm{E}-05$ & 82,25 & 6,04 & 89,31 & 8,69 & 96,34 & 11,96 \\
\hline
\end{tabular}

transition frequency and Fig. 6 shows the 5- and 3-days cumulative rainfall events. Observing the results it is possible to note that the optimal models provide more accurate results above all those concerning the crosscorrelation values.

Another purpose of this study case regards the number of parameters. The Vector $\operatorname{Ar}(\mathrm{p})$ model is characterized by $p \times n^{2}$ parameters, for instance a simple $\operatorname{Var}(2)$ applied on 5 series needs 50 parameters. To overcome this limit they can be reduced by fixing no-significant parameters to zero. Following the T-ratio approach, a SVar(1) with 6 parameters and a $S \operatorname{Var}(2)$ with 14 parameters are defined and then estimated with EGLS method (Lutkepohl, 1993). Looking at the obtained results in Table 1, Figs. 4, 5 and 6 it is evident that these simple models statistically lose information but also that they are able to generate usable daily rainfall synthetic series. 

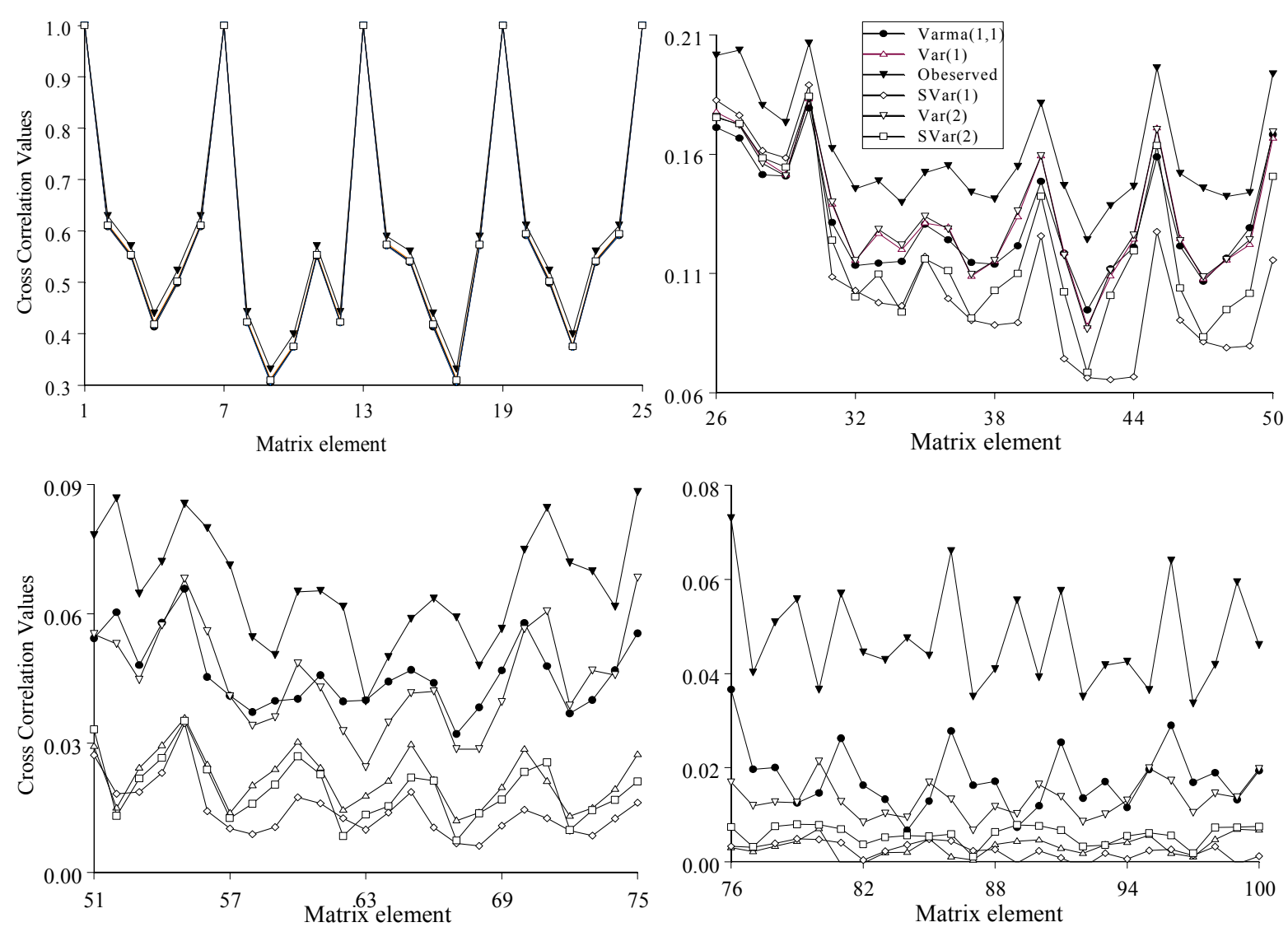

Fig. 4. Crosscorrelation-matrix representation related to $\operatorname{Var}(1), \operatorname{Var}(2), S \operatorname{Var}(1), S \operatorname{Var}(2)$, $\operatorname{Varma}(1,1)$. Boxes show: (a) 25 values of crosscorrelation matrix at lag=0; (b) 25 values of crosscorrelation matrix at lag=1 (c) 25 values of crosscorrelation matrix at lag=2 (d) 25 values of crosscorrelation matrix at lag $=3$.

\section{Conclusion}

In this paper the multivariate linear parametric modelling is applied on 5 daily rainfall time series. The first aim of the application is to test this approach then examine the differences among several models like: $\operatorname{Var}(1)$, optimal $\operatorname{Var}(\mathrm{p})$ and $\operatorname{Varma}(\mathrm{p}, \mathrm{q})$. The described case study shows that this modelling can simulate rainfall time series and that the optimal models prove to be favourable instead of the simplest $\operatorname{Var}(1)$. The limit of these models is the number of the parameters. It frequently happens to have cases with 50 or 70 parameters to estimate. The case study highlights that the Subset Var models can reduce the number of the parameters keeping the results good for hydrological application.

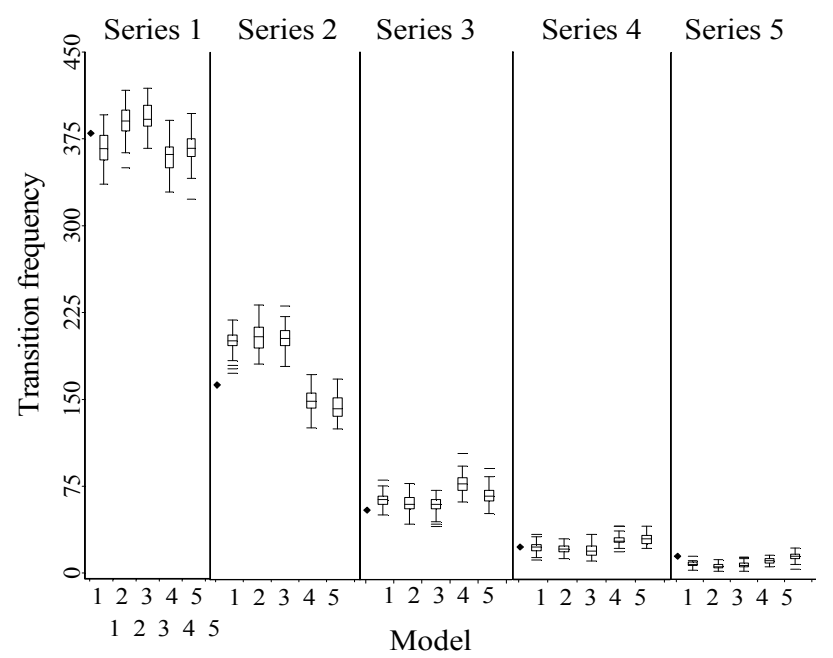

Fig. 5. Transition Frequency of 5 days estimated on each of the observed series (black point) and on the 50 simulated series using a $\operatorname{Var}(1)$ [1], $S \operatorname{Var}(1)$ [2],$S \operatorname{Var}(2)$ [3], $\operatorname{Var}(2)$ [4], $\operatorname{Varma}(1,1)$ [5]. 


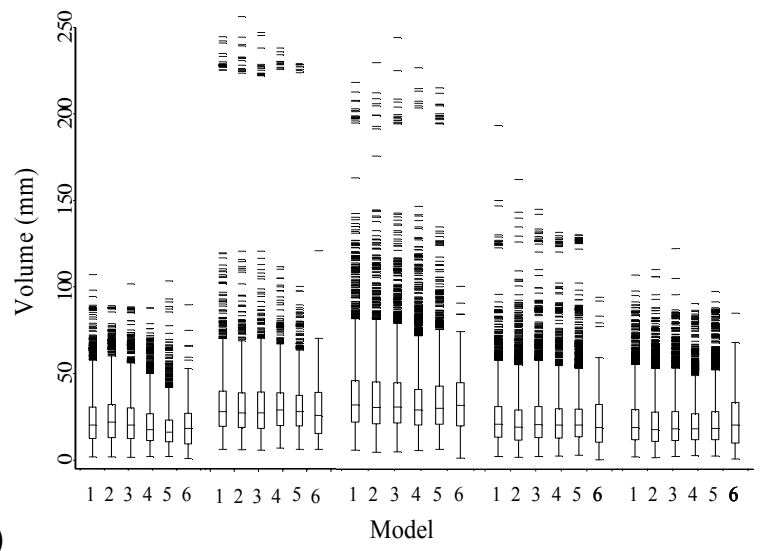

(a)

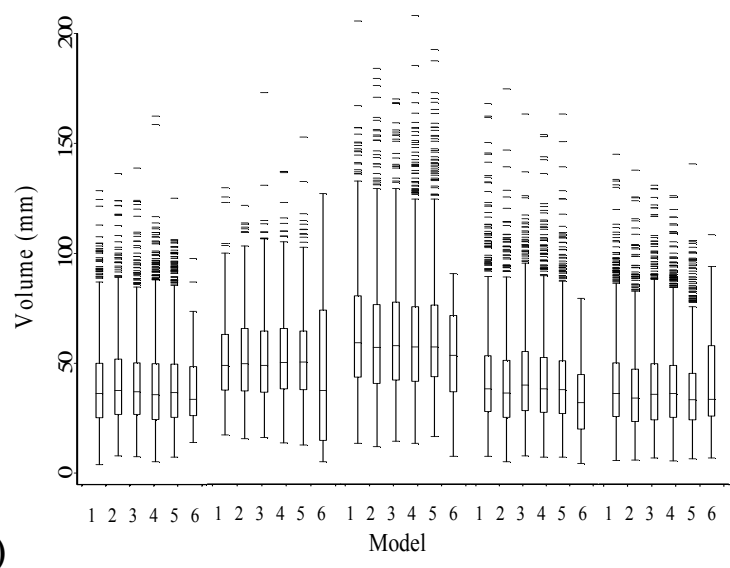

Fig. 6. Volume box plots of 3- (a) and 5- (b) days events. Each block of six box-plots is referred to a series. The box-plots 1,2,3,4 and 5 are referred to the 50 simulated series obtained with $\operatorname{Var}(1)$ [1], SVar(1) [2] , SVar(2) [3], Var(2) [4], Varma(1,1) [5]. The box plot 6 is referred to the observed series. The box-plots are characterized by the median, the $25 \%$ and $75 \%$ interquartile and the outliers.

Acknowledgements. The authors thanks R. Rudari for rainfall data and for precious comments on obtained results. This work was supported by CNR-GNDCI.

Edited by: L. Ferraris Reviewed by: A. Cancelliere and other referees

\section{References}

Box, G. E. P. and Cox, D.: An analysis of transformation (with discussion), Journal of the Royal Statistical Society, B, 26, 211246, 1964.

Grimaldi, S.: Linear parametric models applied on daily hydrological series, Journal of Hydrologic Engineering, 9, 5, 383-391, 2004.

Grimaldi, S., Tallerini, C., and Serinaldi, F.: "Modelli multivariati lineari per la generazione di serie di precipitazioni giornaliere", Proceedings, Giornata di Studio: Metodi Statistici e Matematici per l'Analisi delle Serie Idrologiche, Napoli, maggio 2004.

Hipel, K. W. and McLeod, A. I.: Time Series Modelling of Water Resources and Environmental Systems, Elsevier Science, 1994.

Hosking, J. R. M.: The Multivariate Portmanteau Statistic, Journal of the American Statistical Association, 75, 371, 502-608, 1980.

Hall, A. D. and Nicholls, D. F.: The Exact Maximum Likelihood Function of Multivariate Autoregressive Moving Average Models, Biometrika, 66, 259-264, 1979.

Lutkepohl, H.: Introduction to Multiple Time Series Analysis, 2nd Edition, Springer-Verlag, Berlin, 1993.

Rudari, R.: Predicibilità del clima europeo ed influenze delle forzanti a scala sinottica su eventi regionali di precipitazione intensa”, PhD Thesis, manuscript in Italian, 2001.

Shea, B. L.: The exact likelihood of a Vector Autoregressive Moving Average model, Applied Statistics, 38(1), 161-184, 1989.

Trindade, A., Brockwell, P. J., and Dahlhaus, R.: Modified Burg Algorithms for Multivariate Subset Autoregression, Technical Report 2002-015, Department of Statistics, University of Florida, 2002.

Whille, P.: On the fitting of multivariate autoregressions, and the approximate canonical factorization of spectral density matrix, Biometrika, 50, 1/2, 129-134, 1963. 